\title{
Impact of energy-transfer-upconversion on the performance of hybrid Er:YAG lasers
}

\author{
Ji Won Kim, J. K. Sahu and W. A. Clarkson \\ Optoelectronics Research Centre, University of Southampton, Southampton, SO17 1BJ, UK
}

\begin{abstract}
Using a hybrid fiber-bulk laser scheme based on Er:YAG, we have achieved $\sim 60 \mathrm{~W}$ and $\sim 30 \mathrm{~W}$ of continuous-wave output at $1645 \mathrm{~nm}$ and $1617 \mathrm{~nm}$ respectively, and Q-switched pulse energies up to $\sim 30 \mathrm{~mJ}$ (limited by coating damage). Investigation of various factors influencing laser performance has revealed that energy-transfer-upconversion can have a very detrimental impact on efficiency, even in continuous-wave mode of operation. In this paper we report on the results of this study, discuss various measures for reducing energy-transfer-upconversion and its effect on laser performance, and consider the prospects for further increase in output power and pulse energy.
\end{abstract}

\section{Introduction}

Erbium-doped solid-state laser sources operating in the eyesafe wavelength regime around 1.5-1.6 $\mu \mathrm{m}$ have applications in a number of areas including free-space communications, remote sensing and ranging. For many of these applications, the requirement for high output power and/or high pulse energy is often accompanied by the need for high efficiency and good beam quality. The standard approach for producing laser output in the required $1.5-1.7 \mu \mathrm{m}$ wavelength region is via direct diode pumping of erbium-ytterbium co-doped bulk glass or crystal lasers using diode lasers at 940-980 nm. However, this approach suffers from the problem that a large fraction of the pump power is converted to heat in the bulk laser material resulting in strong thermal lensing, which can severely degrade laser beam quality and efficiency, and thermally-induced stress, which can cause catastrophic failure of the bulk material ${ }^{1}$. This problem can be avoided by using a cladding-pumped fiber laser configuration. Fibers benefit from a geometry which allows simple thermal management and hence offer a high degree of immunity from the thermal effects which are so detrimental to conventional bulk solid-state lasers. Recent progress in scaling output power from cladding-pumped Er, Yb fiber lasers has been dramatic with maximum reported power levels exceeding $100 \mathrm{~W}^{2}$. Unfortunately, due to their long device lengths and small core size, fiber lasers suffer from detrimental nonlinear effects, especially when operating in the high peak power pulsed regime, which can limit efficiency. Furthermore, pulse energies are limited by amplified spontaneous emission and by damage to the fiber facets. For this reason, attention has focussed on resonant (in-band) pumping of Erdoped bulk crystal lasers using direct diode pumping at $1470 \mathrm{~nm}$ or $1532 \mathrm{~nm}^{3,4}$ or, alternatively, a hybrid fiber-bulk laser configuration $^{5}$. The former approach has the attraction of simplicity, but suffers from the drawback that the currently available InGaAsP/InP diode pump lasers have relatively broad linewidths $(\sim 10 \mathrm{~nm})$ and poor beam quality, which impose severe constraints on the choice crystal, doping level and resonator configuration, limiting flexibility and making it difficult to achieve high efficiency at room temperature. The hybrid laser approach is requires an intermediate (fiber) laser stage as a brightness and wavelength converted, but offers very high overall efficiency and flexibility in choice of bulk gain medium, resonator design and mode of operation.

The rationale behind the hybrid laser approach is to combine advantages of cladding-pumped fiber lasers for efficient cw high-power generation with the energy storage and high pulse energy capabilities of bulk solid-state lasers. This has the important advantage that most of the heat generated via quantum defect heating (typically 40 \%) is deposited in the fiber, with only $\sim 6-7 \%$ of the fiber laser output power converted to heat in the bulk crystal. The net result is that thermal effects in the bulk Er laser are dramatically reduced leading to the prospect of much improved efficiency, beam quality and higher output power. The output beam quality from the fibre laser is determined by the waveguiding properties of the active-ion-doped core, which can easily be tailored to produce a single-spatial-mode output beam with little, if any, impact on beam quality due to thermal lensing. Also, by employing wavelengthdependent feedback provided by an in-fiber Bragg grating or by an external cavity containing a simple diffraction grating, the Er, Yb fiber laser's wavelength can be tuned to precisely coincide with the strongest absorption line in the Er-doped bulk crystal (i.e. $1532 \mathrm{~nm}$ in Er:YAG). Using this hybrid laser scheme, we have achieved $\sim 60 \mathrm{~W}$ and $\sim 30 \mathrm{~W}$ of continuous-wave output at $1645 \mathrm{~nm}$ and $1617 \mathrm{~nm}$ respectively, and Q-switched pulse energies up to $\sim 30 \mathrm{~mJ}$ (limited by coating damage) ${ }^{5-7}$. 
However, investigation of various factors influencing laser performance has revealed that energy-transferupconversion (ETU) can have a very detrimental impact on efficiency, even in continuous-wave mode of operation. ETU is the process by which energy is transferred between two neighbouring excited ions (donor ion to acceptor ion). In these case of Er:YAG (see Fig. 1), energy is transferred between neighbouring ions in the upper laser level, ${ }^{4} \mathrm{I}_{13 / 2}$, and the acceptor is excited to a higher energy level, ${ }^{4} \mathrm{I}_{9 / 2}$, whilst the donor decays to a lower laser level, ${ }^{4} \mathrm{I}_{15 / 2}$. The acceptor ion then decays non-radiatively (via multi-phonon relaxation) back to the upper laser level. Hence ETU converts two excited ions into one excited ion plus extra heat, resulting in a dramatic reduction in the effective upper laser level lifetime and a large increase in the fractional heat loading. The net result can be a significant degradation in laser performance, particularly in pulsed (Q-switched) lasers or in more complicated (higher loss) cw laser configurations requiring a high upper-laser-level population ${ }^{8-10}$. Thus, an accurate knowledge of the upconversion parameter and its impact on laser performance is very important for power scaling of Er-doped solid-state lasers.

In this paper we present the results of a preliminary study of the impact of energy-transfer-upconversion on the performance of hybrid lasers based on Er:YAG in continuous-wave mode of operation. We show that very low $\mathrm{Er}^{3+}$ concentrations $(<0.25$ at.\%) are required to render the impact of ETU negligible in certain operating regimes. We also consider the prospects for further increase in output power and pulse energy.

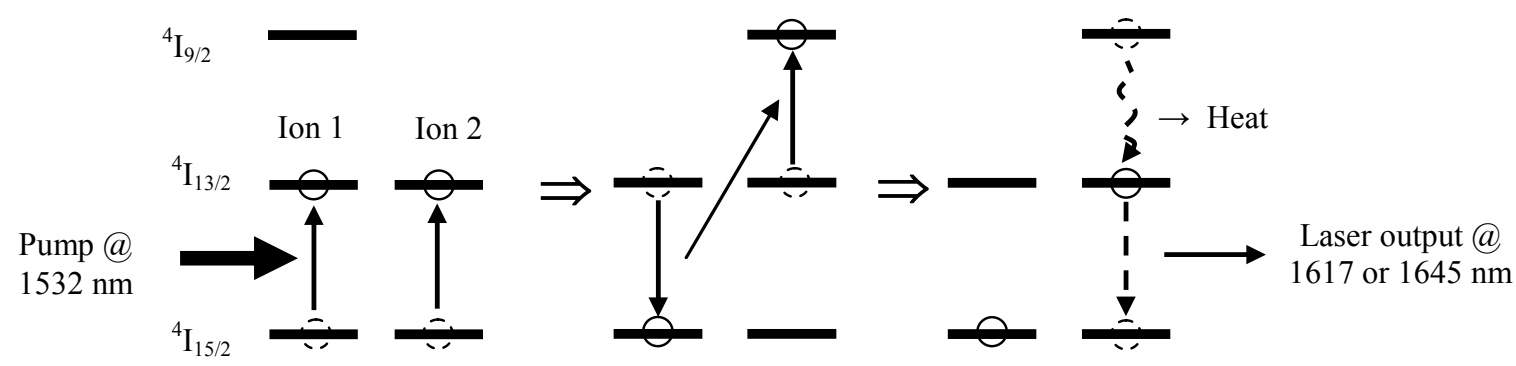

Fig. 1. Energy-transfer-upconversion in Er:YAG.

\section{Experiment}

The experimental set-up used to investigate the influence of ETU on laser performance in Er:YAG is shown in Fig. 2. The main purpose of our study was to see how the choice $\mathrm{Er}^{3+}$ doping level affects the performance. The loss of excitation due to ETU depends on the ETU rate parameter and excitation density in the upper laser level, both of which are dependent on the doping level. To perform this study, we employed a simple two-mirror resonator design for the Er:YAG laser comprising a plane input coupler mirror (IC) with high reflectivity $(>99.8 \%)$ at the lasing wavelength of $1645 \mathrm{~nm}$ and high transmission $(>98.0 \%$ ) at the pump wavelength of $1532 \mathrm{~nm}$ and a concave mirror (OC) with reflectivity of $95 \%$ at $1550 \sim 1650 \mathrm{~nm}$. The latter served as the output coupler and also ensured that unabsorbed pump light was incident on the Er:YAG crystal for a second time. Pump light was provided by a cladding-pumped Er,Yb fiber laser (EYDFL) constructed in-house (see Fig. 2). The employed an Er,Yb double-clad fiber (EYDF) with an Er,Yb codoped phospho-silicate core of $30 \mu \mathrm{m}$ diameter and $0.22 \mathrm{NA}$, surrounded by a pure silica D-shaped inner-cladding of 400 $\mu \mathrm{m}$ diameter with a calculated NA of 0.49 . The effective absorption coefficient for pump light at $975 \mathrm{~nm}$ (launched into the inner-cladding) was measured to be $\sim 7.1 \mathrm{~dB} / \mathrm{m}$, and hence a relatively short fiber length of $\sim 2.5 \mathrm{~m}$ was selected for our experiment. Both end sections of the fiber were carefully mounted in water-cooled V-groove heat sinks maintained at $17{ }^{\circ} \mathrm{C}$ to prevent thermal damage to the fiber coating due to unlaunched pump power and heat generated in the core due to quantum defect heating. Pump power was provided by two $975 \mathrm{~nm}$ nine-diode-bar pump modules, which were polarisation-combined and then the resulting beam was split into two beams of roughly equal power to allow pumping of the EYDF from both ends to spread the heat load. Pump light was launched into the fiber ends using dichroic mirrors with high reflectivity $\left(>99.5 \%\right.$ at $\left.45^{\circ}\right)$ at the pump wavelength, and high transmission $(>98 \%)$ at $1530-1570 \mathrm{~nm}$ to allow extraction of the signal beam. Feedback for lasing was provided by a perpendicularly-cleaved facet at the output end of 


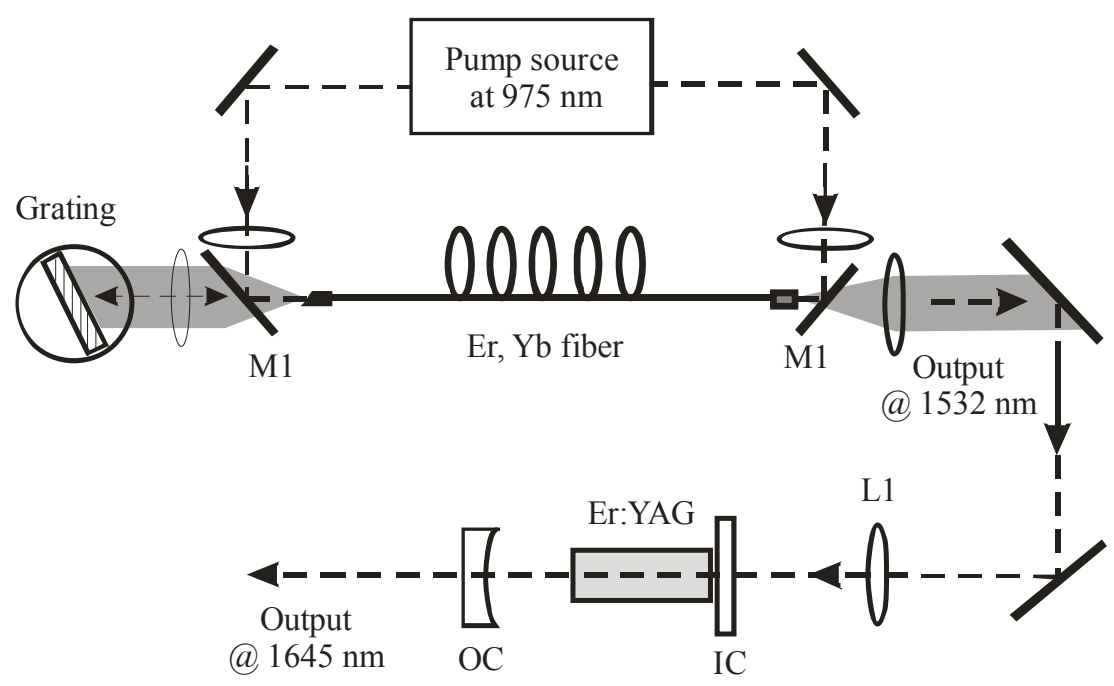

Fig. 2. Schematic diagram of the Er,Yb fiber laser and the Er:YAG laser resonator.

the fiber and, at the opposite end, by a simple external cavity comprising an $120 \mathrm{~mm}$ focal length collimating lens and the replica diffraction grating $(600$ lines $/ \mathrm{mm}$ ), which was aligned in the Littrow configuration to provide wavelength selective feedback for adjusting the lasing wavelength. The fiber end facet nearest the grating was angle-polished at $\sim 14^{\circ}$ to suppress parasitic lasing between the two fiber end facets. Using this configuration, the EYDFL produced up to $120 \mathrm{~W}$ of output at $1532 \mathrm{~nm}$ in a beam with $\mathrm{M}^{2}<5$ for $\sim 440 \mathrm{~W}$ of launched pump power (Fig. 3).

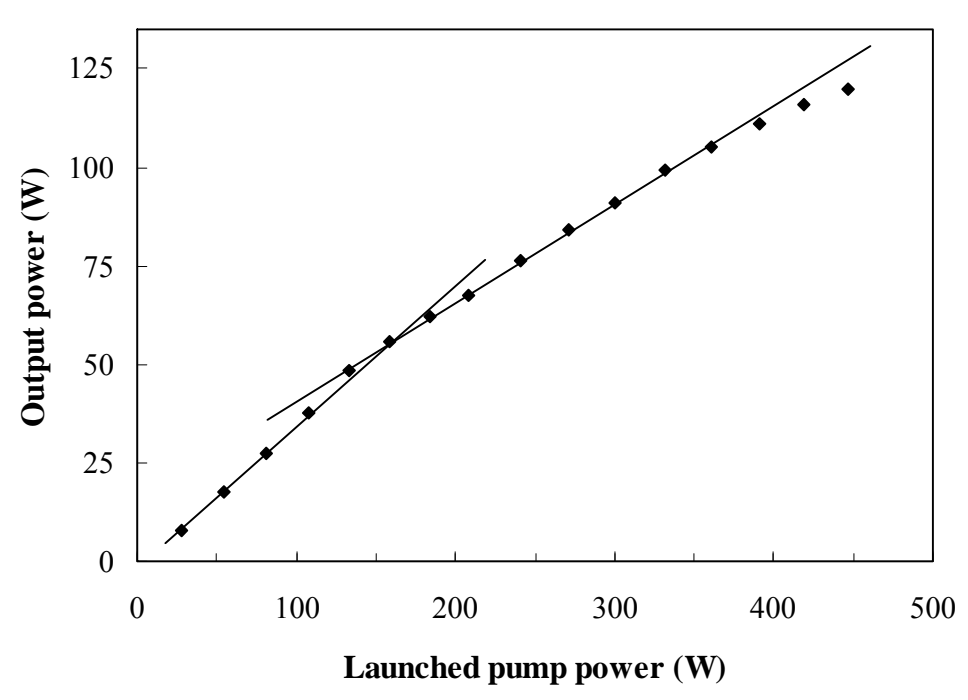

Fig. 3. Er,Yb fiber laser output power at $1532 \mathrm{~nm}$ versus launched pump power.

The pump beam from the EYDFL was focussed to a beam waist radius of $180 \mu \mathrm{m}$ inside the Er:YAG crystal and the radius of curvature of concave output coupler $(100 \mathrm{~mm})$ and cavity length $(15-50 \mathrm{~mm})$ were selected to give a matched 
$\mathrm{TEM}_{\mathrm{oo}}$ radius depending on the strength of thermal lensing in the laser rod. Er:YAG rods with five different doping levels were tested in the laser resonator. The doping levels $(4.0$ at $\%, 2.0$ at.\%, 1.0 at.\%, 0.5 at.\% and 0.25 at.\%) and corresponding rod lengths $(3.5 \mathrm{~mm}, 7.0 \mathrm{~mm}, 15 \mathrm{~mm}, 29 \mathrm{~mm}$ and $58 \mathrm{~mm})$ were selected so that the fraction of pump light absorbed for a single-pass of the pump light was $\sim 98 \%$ and hence the same for each rod. The corresponding absorption coefficient at $1532 \mathrm{~nm}$ for a 1.0 at.\% Er doping level is $1.4 \mathrm{~cm}^{-1}$. The end faces of each rod were antireflection coated over the range 1530-1650 $\mathrm{nm}$ (i.e. at the pump and lasing wavelengths) and the rods were mounted in water-cooled aluminium heat-sinks maintained a temperature of $17^{\circ} \mathrm{C}$ positioned in close proximity to the plane mirror (IC). In each case the laser performance (i.e. threshold pump power and laser output power as a function of pump power) was measured. The threshold pump power was determined as the power required for the onset of relaxation oscillation, detected using an InGaAs photodetector and an oscilloscope.

\section{Results and discussion}

Table 1 shows the measured threshold pump power and slope efficiency for the Er:YAG lasers with different Er concentrations. For low Er concentration levels (i.e. 0.25 at.\% and 0.5 at.\%) the threshold pump is approximately the same at just over $1 \mathrm{~W}$. However, for higher Er concentration levels there is a dramatic increase in threshold pump power to $\sim 7.8 \mathrm{~W}$ for the 4.0 at.\% doping level. There is also a marked decrease in slope efficiency at higher doping levels. In the case of the 4.0 at.\%, lasing was terminated at slightly higher power than threshold, suggesting a much higher fractional heat loading than for crystals with lower doping levels. It should be stressed the Er:YAG resonator is not designed for high efficiency as the output coupling transmission is too low and the rod length too long, and hence with a modified design much higher power levels and efficiencies can be achieved at low doping levels ${ }^{6}$. The purpose of this study is a comparison between Er:YAG rods with different doping levels under otherwise identical conditions. The results summarised in Table 1 show very clearly that there is a strong dependence of laser performance at $1645 \mathrm{~nm}$ on doping level under cw operating conditions.

\begin{tabular}{|c|c|c|c|c|c|}
\hline & $0.25 \%$ Er:YAG & $0.5 \%$ Er:YAG & $1.0 \%$ Er:YAG & $2.0 \%$ Er:YAG & $4.0 \%$ Er:YAG \\
\hline $\begin{array}{c}\text { Threshold pump power } \\
\text { (W) }\end{array}$ & 1.05 & 1.1 & 1.56 & 3.7 & 7.8 \\
\hline Slope efficiency (\%) & 36 & 34 & 19 & 13 & - \\
\hline
\end{tabular}

Table 1. Threshold pump power and slope efficiency of Er:YAG crystals with different Er concentrations.

The standard expression for threshold pump power, $\mathrm{P}_{\text {th }}$ for a quasi-three-level laser is ${ }^{11}$

$$
P_{t h}=\frac{\pi h v_{p}}{2 f_{b} \eta_{p} \tau}\left(w_{p}^{2}+w_{0}^{2}\right)\left(\frac{\delta}{2 \sigma}+f_{a} N_{0} L\right)
$$

where $v_{p}$ is the laser frequency, $\eta_{p}$ is the pump quantum efficiency, $\tau$ is the fluorescent lifetime, $w_{p}$ and $w_{0}$ are the pump and laser mode radii respectively, $\delta$ is the round-trip cavity loss including output coupler transmission, $\sigma$ is the emission cross section, $N_{0}$ is the active ion population, $L$ is the length of the laser rod and, $f_{a}$ and $f_{b}$ are the fractional populations in the lower and upper laser levels respectively. The values for $N_{0} L, w_{p}, w_{0}$, and $\delta$ are the same for each laser studied, so, neglecting the temperature dependence of the thermal occupancy factors, $f_{a}$ and $f_{b}$, should yield the same values for threshold pump power. Using $f_{b}=0.211, f_{b}=0.0199, \tau=6 \mathrm{~ms}, w_{0}=w_{p}=180 \mu \mathrm{m}, \sigma=1.9 \times 10^{-20} \mathrm{~cm}^{2}, N_{0} L=2.1 \times 10^{20}$ $\mathrm{cm}^{-2}$ and $\delta=0.1$, then from equation (1) we calculate the threshold pump power as $\sim 0.8 \mathrm{~W}$. This is in reasonable agreement with the measured threshold power for the 0.25 at.\% and 0.5 at.\% Er concentrations, but not at higher concentration levels.

Recently, we reported on an analytical model for threshold pump power in the quasi-three-level laser taking into account the effect of ETU ${ }^{12}$. The resulting modified expression for threshold pump power is 


$$
P_{t h}=\frac{h v_{p} \pi w_{p}^{2} \delta}{2 \sigma \tau \eta_{p}}\left[\frac{a^{2} W_{u p} \alpha_{p} \tau \delta}{4 \sigma}+Q+\frac{\alpha_{p} L(K-Q)}{2}\right]+\frac{h v_{p} \pi w_{p}^{2} L(K-Q)}{2 a^{2} \tau^{2} W_{u p} \eta_{p}}\left[Q+\frac{\alpha_{p} L(K-Q)}{4}\right]
$$

where $\alpha_{p}$ is the effective absorption coefficient for the pump, $W_{\text {up }}$ is the upconversion parameter, $a=1 /\left(f_{a}+f_{b}\right), b=f_{a} /\left(f_{a}+f_{b}\right)$, $K=a+2 a b \tau W_{u p} N_{t}$, and $Q^{2}=K^{2}-4 a^{2} \tau^{2} W_{u p}\left(\frac{b}{\tau} N_{t}+b^{2} W_{u p} N_{t}^{2}\right)$. Using equation (2) and by measuring the increase in threshold pump power as a function of cavity loss in a modified resonator design, we have determined the upconversion parameter for a 1.0 at.\% Er:YAG crystal to be $6.1 \times 10^{-18} \mathrm{~cm}^{3} / \mathrm{s}$. This value is in good agreement with the previously reported value determined in the standard way (i.e. via measurement of fluorescence decay times). If we assume that the ETU parameter is scales linearly with doping concentration ${ }^{13}$, then from equation (2) we can calculate the threshold pump power as a function of Er concentration. The results (plotted in Fig. 4) indicate that the trend is in reasonably good agreement with that observed experimentally. For comparison Fig. 4 also show a plot of the predicted threshold pump power as a function of doping level in the absence of ETU, but including the temperature dependence of the thermal occupancy factors (i.e. $f_{a}$ and $f_{b}$ ) assuming quantum defect heating is the sole source of heat generation. It can be seen that under our experimental operating conditions there is a negligible increase in three-level character due to the increase in thermal loading density with higher doping levels. This serves once again to confirm the importance of the role of ETU on laser performance.

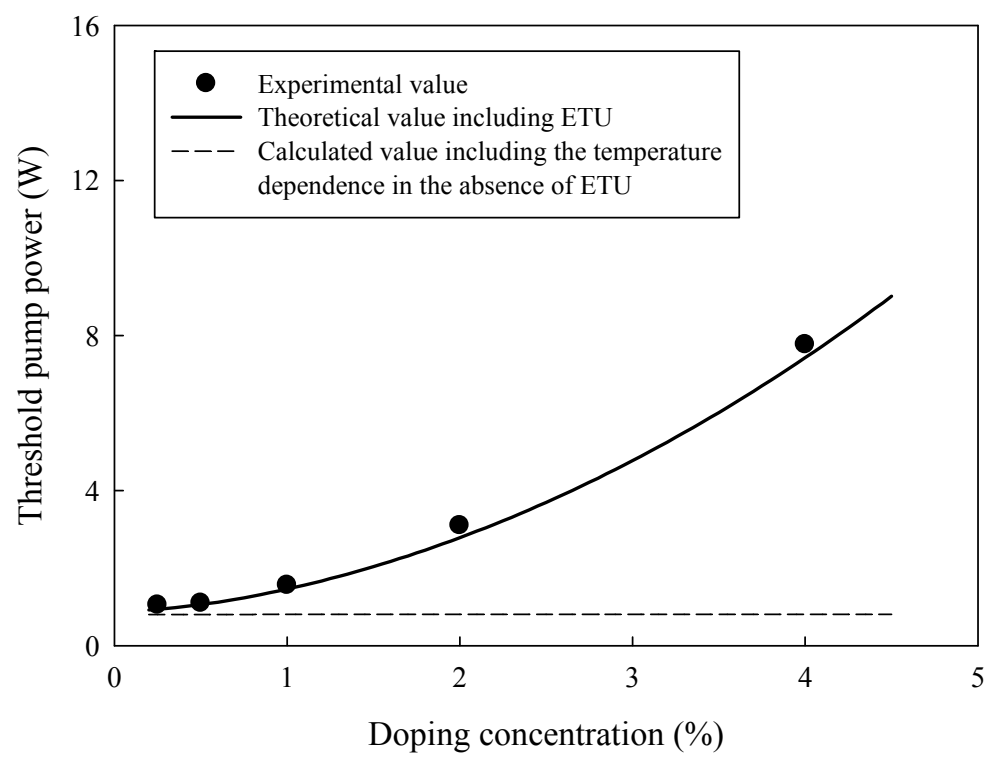

Fig. 4. Experimental (open circle) and calculated threshold pump power (solid-line) as a function of a doping concentration. The dashed line shows the effect of quantum defect heating on threshold pump power in the absence of ETU.

Fig.5 shows Er:YAG laser output power at $1645 \mathrm{~nm}$ versus pump power for 2.0 at.\%, 1.0 at.\%, 0.5 at.\% and 0.25 at.\% doping levels. It can be seen that in addition to the increase in threshold pump power with doping level, there is a very marked decrease in output power and slope efficiency. This is partly due to the higher threshold pump power and hence operation at a maximum pump power that is fewer times the threshold pump power. However, there is also a contribution due to thermal effects (i.e. aberrated thermal lensing) due to the extra heat generated by ETU. These results emphasize the need to use low $\mathrm{Er}^{3+}$ doping levels for high efficiency even when operating in cw mode. 


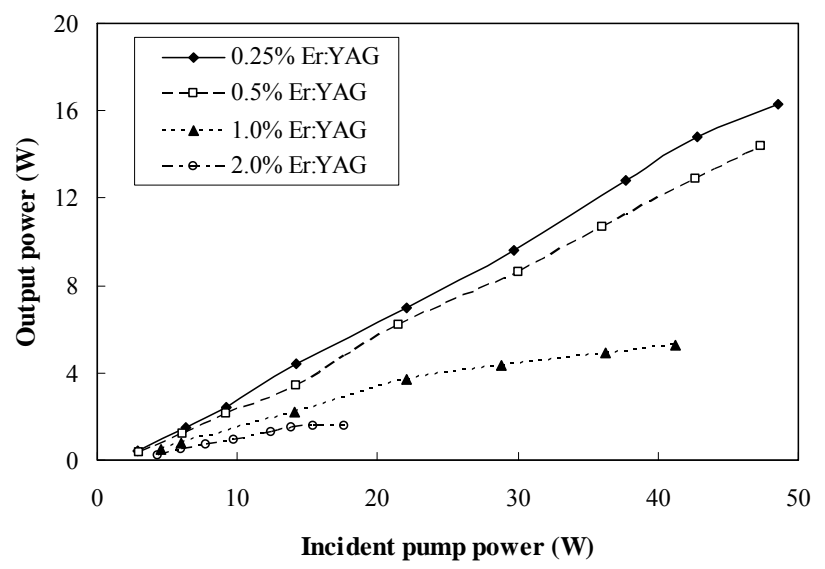

Fig. 5. Er:YAG laser output power as a function of incident pump power for different $\mathrm{Er}^{3+}$ doping levels.

We have also investigated operation on the $1617 \mathrm{~nm}$ line. This transition terminates in a higher lying Stark level than the $1645 \mathrm{~nm}$ transition and hence has a much more pronounced three-level character requiring $\sim 14.6 \%$ of the Er ions to be excited to the upper laser level manifold to reach transparency. Thus, operation at $1617 \mathrm{~nm}$ requires a resonator design with additional wavelength discrimination to suppress the $1645 \mathrm{~nm}$ line or a much higher output coupled transmission. The resonator used in our experiments (shown in Fig. 6) employed a simple four mirror-folded cavity comprising a plane input coupler (IC) with high reflectivity $(>99.8 \%)$ at the lasing wavelength of $1645 \mathrm{~nm}$ and

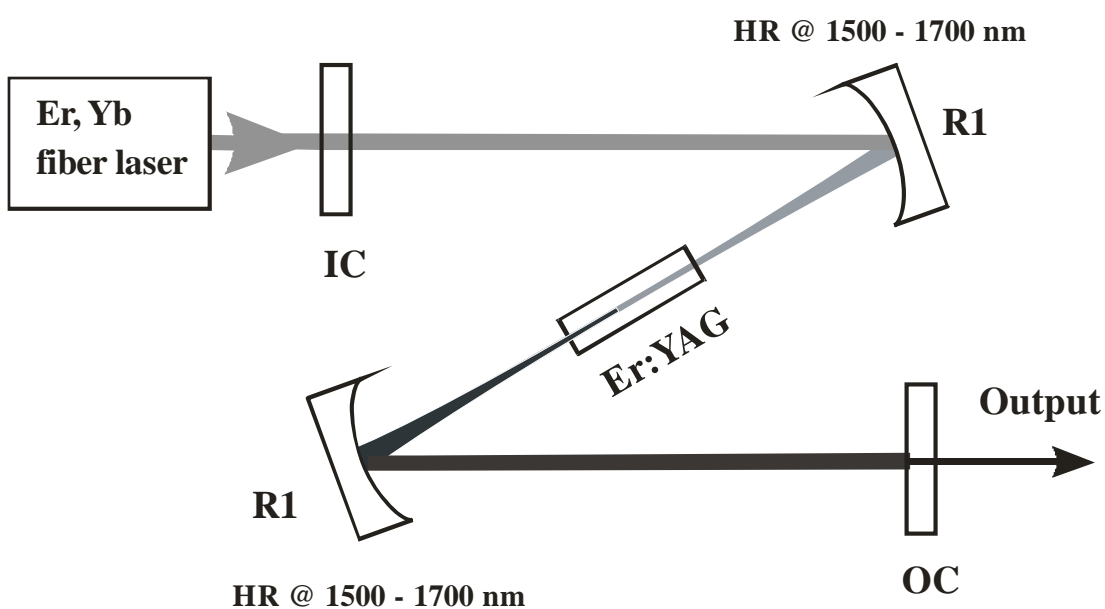

Fig. 6. Schematic diagram of the Er:YAG resonator design for operation at $1617 \mathrm{~nm}$. IC : input coupler mirror with $\mathrm{AR}$ at 1532 $\mathrm{nm}$ and HR at 1600-1700 $\mathrm{nm}$. OC : output coupler mirror with a transmission (T) of 50\% at 1600-1700 nm.

high transmission ( $>98.0 \%$ ) at the pump wavelength of $1532 \mathrm{~nm}$, two concave mirrors (R1) with radius of curvature $100 \mathrm{~mm}$ and high reflectivity at the lasing wavelength and a plane output coupler with a relatively high transmission of $50 \%$ at the lasing wavelength. Laser performance was investigated for Er:YAG rods with Er doping levels of 1.0 at.\%, 0.5 at.\% and 0.25 at.\% and corresponding rod lengths $15 \mathrm{~mm}, 29 \mathrm{~mm}$ and $58 \mathrm{~mm}$. We were unable to achieve lasing using rods with higher doping levels. Figure 7 shows the laser output power versus incident pump power for the three doping levels. It can be seen that laser performance is more strongly dependent on the Er doping level than for $1645 \mathrm{~nm}$ 
operation with very pronounced roll-over in output power for 1.0 at.\% and 0.5 at.\% doping levels. Only the 0.25 at. $\%$ doping level shows no sign of a power roll over, yielding a maximum output power of $\sim 26 \mathrm{~W}$ for $72 \mathrm{~W}$ of pump power. We attribute the difference in performance for the different Er doping levels to ETU, which, due to the need for a higher excitation density to reach threshold than was the case for $1645 \mathrm{~nm}$ operation, has a much more severe impact on laser performance. The roll-over in power is due to the increased thermal loading which results from ETU.

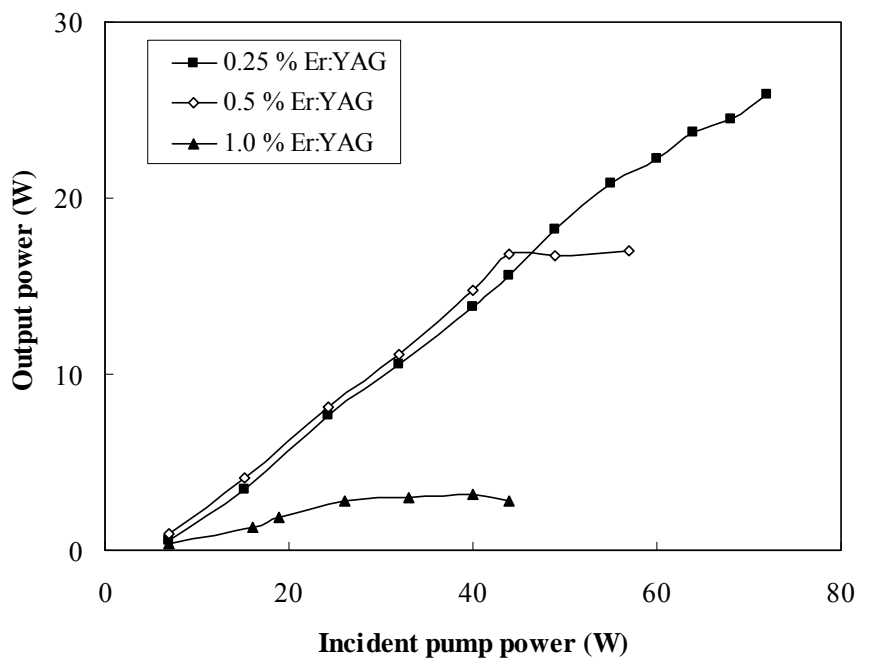

Fig. 7. Er:YAG laser output power at $1617 \mathrm{~nm}$ versus incident pump power.

\section{Conclusion}

We have studied the effect of energy-transfer-upconversion on the performance of hybrid (fibre-laser-pumped) Er:YAG lasers with different Er doping levels. Our results shown that upconversion can have a very detrimental impact on performance (especially for operation on the $1617 \mathrm{~nm}$ line) unless a very low Er doping level (i.e. $<0.25$ at.\%) is used even in continuous-wave mode of operation. This is a surprising result and serves to further confirm the benefits of the hybrid laser architecture over direct diode pumping as the latter requires high doping levels due poor pump beam quality.

This work was funded by the Electro-Magnetic Remote Sensing (EMRS) Defence Technology Centre, established by the UK Ministry of Defence.

\section{Reference}

1. V. I. Zhekov, T. M. Murina, A. M. Prokhorov, M. I. Studenikin, S. Georgescu, V. Lupei, and I. Ursu, “Cooperative process in $\mathrm{Y}_{3} \mathrm{Al}_{5} \mathrm{O}_{12}: \mathrm{Er}^{3+}$ crystals", Sov. J. Quantum Electron. 16, 274 (1986).

2. Y.Jeong, S.Yoo, C.A.Codemard, J.Nilsson, J.K.Sahu, D.N.Payne, R.Horley, P.W.Turner, L.M.B.Hickey, A.Harker, M.Lovelady, A.Piper, "Erbium:ytterbium co-doped large-core fiber laser with 297W continuous-wave output power", IEEE J. Sel. Top. Quantum Electron. 13, 573 (2007).

3. Dmitri Garbuzov, Igor Kudryashov and Mark Dubinskii, "Resonantly diode laser pumped 1.6- $\mu$ m-erbium-doped yttrium aluminium garnet solid-state laser", Appl. Phys. Lett. 86, 131115 (2005).

4. Dmitri Garbuzov, Igor Kudryashov and Mark Dubinskii, "110 W (0.9 J) pulsed power from resonantly diode-laserpumped 1.6 $\mu \mathrm{m}$ Er:YAG laser”, Appl. Phys. Lett. 87, 121101 (2005).

5. D. Y. Shen, J. K. Sahu and W. A. Clarkson, "Highly efficient in-band pumped Er:YAG lasers with $>60 \mathrm{~W}$ of output at 1645 nm", Opt. Lett 31, 754 (2006).

6. D. Y. Shen, J. K. Sahu and W. A. Clarkson, "Electro-optically Q-switched Er:YAG laser in-band pumped by an Er Yb fiber laser", Advanced Solid-State Photonics 2006 Nevada WD4 (2006). 
7. J. W. Kim, J. K. Sahu, W. A. Clarkson, “High power in-band pumped Er:YAG laser at 1617 nm”, CLEO/EuropeIQEC 2007 Munich CA6-4-TUE, (2007).

8. P. J. Hardman, W. A. Clarkson, G. J. Friel, M. Pollnau, D. C. Hanna, "Energy-transfer upconversion and thermal lensing in high-power end-pumped Nd:YLF laser crystals", IEEE Journal of Quantum Electronics 35, 647, (1999).

9. C. Jacinto, S. L. Oliveira, T. Catunda, A. A. Andrade, J. D. Myers, "Upconversion effect on fluorescence quantum efficiency and heat generation in $\mathrm{Nd}^{3+}$-doped materials", Opt. Express, 13, 2040 (2005).

10. S. Bjurshagen, R. Koch, "Modeling of energy-transfer upconversion and thermal effects in end-pumped quasi-threelevel lasers”, Appl. Opt. 43, 4753 (2004).

11. T. Y. Fan, R. L. Byer, "Modeling and cw operation of a quasi-three-level $946 \mathrm{~nm}$ Nd:YAG laser", IEEE J. Quantum Electron. 23, 605 (1987).

12. J. W. Kim, I. O. Musgrave, M. J. Yarrow, W. A. Clarkson, "Simple technique for measuring the energy-transferupconversion parameter in solid-state laser materials", CLEO/Europe 2007 Munich CA-40-MON (2007).

13. M. O. Iskandarov and A. A. Nikitichev, A. I. Stepanov, "Quasi-two-level Er: $\mathrm{Y}_{3} \mathrm{Al}_{5} \mathrm{O}_{12}$ laser for the $1.6 \mu \mathrm{m}$ range", J. Opt. Technol. 68, 885 (2001). 